\title{
The Non-Constant-Sum Colonel Blotto Game
}

\author{
BRIAN ROBERSON \\ DMITRIY KVASOV
}

CESIFO WORKING PAPER NO. 2378

CATEgORY 2: PUBliC CHOICE

August 2008

Presented at CESifo Venice Summer Institute 2008, Workshop on

'ADVANCES IN THE THEORY OF CONTESTS AND ITS APPLICATIONS'

An electronic version of the paper may be downloaded

- from the SSRN website:

www.SSRN.com

- from the RePEc website:

- from the CESifo website:

www.RePEc.org

www.CESifo-group.org/wp 


\title{
The Non-Constant-Sum Colonel Blotto Game
}

\begin{abstract}
The Colonel Blotto game is a two-player constant-sum game in which each player simultaneously distributes her fixed level of resources across a set of contests. In the traditional formulation of the Colonel Blotto game, the players' resources are "use it or lose it" in the sense that any resources which are not allocated to one of the contests are forfeited. This paper examines a non-constant-sum version of the Colonel Blotto game which relaxes this use it or lose it feature. We find that if the level of asymmetry between the players' budgets is below a threshold, then the unique set of equilibrium univariate marginal distributions of the non-constant-sum game is equivalent up to an affine transformation to the unique set of equilibrium univariate marginal distributions of the constant-sum game. Once the asymmetry of the players' budgets exceeds the threshold we construct a new equilibrium.
\end{abstract}

JEL Code: C7.

Keywords: Colonel Blotto game, all-pay auction, contests.

\author{
Brian Roberson \\ Miami University \\ Department of Economics \\ Richard T. Farmer School of Business \\ 208 Laws Hall \\ Oxford, OH 45056-3628 \\ USA \\ robersba@muohio.edu
}

\author{
Dmitriy Kvasov \\ University of Auckland \\ Department of Economics \\ Business School \\ Level 1 Commerce A Building \\ 3A Symonds Street \\ Auckland City 1142 \\ New Zealand \\ d.kvasov@auckland.ac.nz
}

We are grateful to Dan Kovenock for very helpful comments. 


\section{Introduction}

Kvasov (2007) introduces a non-constant-sum version of the classic Colonel Blotto game. Originating with Borel (1921), the Colonel Blotto game examines strategic resource allocation across multiple simultaneous contests. Borel formulates this problem as a constant-sum game involving two players, $\mathrm{A}$ and $\mathrm{B}$, who must each allocate a fixed amount of resources, $X_{A}=X_{B}$, over a finite number of contests. Each player must distribute their resources without knowing their opponent's distribution of resources. In each contest, the player who allocates the higher level of resources wins, and the payoff for the whole game is the sum of the wins across the individual contests. A novel feature of the Colonel Blotto game is that a mixed strategy is a multivariate distribution function in which each individual contest is represented as a dimension. The restriction on the players' expenditures implicitly places a constraint on the support of the players' joint distributions. Namely, each point contained in the support of a player's joint distribution must satisfy their budget constraint with probability one.

While a focal point in the early game theory literature, ${ }^{1}$ the Colonel Blotto game has also experienced a recent resurgence of interest (see for example Golman and Page (2006), Hart (2008), Kovenock and Roberson (2007), Laslier (2002), Laslier and Picard (2002), Roberson (2008), or Weinstein (2005)). Most closely related to this paper are Roberson (2006) and Kvasov (2007). For all configurations of the asymmetric Colonel Blotto game with three or more contests, Roberson (2006) provides the characterization of the unique equilibrium payoffs. ${ }^{2}$ The characterization of the equilibrium univariate marginal distributions and the existence of joint distributions which provide the equilibrium univariate marginal distributions and expend the players' respective budgets with probability one are also given in Roberson (2006).

In Borel's original formulation of the Colonel Blotto game the players' resources are "use it or lose it" in the sense that any resources which are not allocated to one of the contests are forfeited. Kvasov's (2007) non-constant-sum version of the Colonel Blotto game relaxes this use it or lose it feature. In the case of symmetric budgets, that paper

1 See Kvasov (2007) or Roberson (2006) for surveys of this literature.

2 The case of $n=2$, with symmetric and asymmetric forces, is discussed by Gross and Wagner (1950). Moving from $n=2$ to $n \geq 3$ greatly enlarges the space of feasible $n$-variate distribution functions, and the equilibrium strategies examined in that paper differ dramatically from the case of $n=2$. 
establishes that a suitable affine transformation of the constant-sum equilibrium is an equilibrium of the non-constant-sum game.

In this paper we extend the analysis of the non-constant-sum version of the Colonel Blotto game to allow for asymmetric budget constraints. As long as the level of asymmetry between the players' budgets is below a threshold, we find that there exists an affine transformation of the equilibrium to the constant-sum game which provides an equilibrium to the non-constant-sum game. Once the asymmetry of the players' budgets exceeds the threshold this correspondence breaks down and we construct an entirely new equilibrium. For all configurations of the players' aggregate levels of force we characterize the unique equilibrium payoffs, and for most parameter configurations we characterize the complete set of equilibrium univariate marginal distributions.

Section 2 presents the model. Section 3 characterizes the equilibrium payoffs and the equilibrium set of univariate marginal distributions for the asymmetric non-constantsum version of the Colonel Blotto game. Section 4 concludes.

\section{The Model}

Two players, $A$ and $B$, simultaneously enter bids in a finite number, $n \geq 3$, of independent all-pay auctions. Each contest has a common value of $v$ for each player. Each player has a fixed level of available resources (or budget), $X_{i}$ for $i=A, B$. Let $X_{A} \leq X_{B}$, and define the modified budgets as $\bar{X}_{A}=\min \left\{X_{A}, n v / 2\right\}$ and $\bar{X}_{B}=\min \left\{X_{B}, \sqrt{n v \bar{X}_{A} / 2}\right\} .{ }^{3}$ In the case that the players enter the same bid in a given contest, it is assumed that player B wins the auction if the common bid is $X_{A}$, otherwise each player wins the auction with equal probability. The specification of the tie-breaking rule does not affect the results as long as $(2 / n) \bar{X}_{B} \leq \bar{X}_{A}$. In the case that $(2 / n) \bar{X}_{B}>\bar{X}_{A}$, this specification of the tie-breaking rule avoids the need to have player $B$ provide a bid arbitrarily close to, but above, player $A$ 's maximal bid, $X_{A}$. A range of tie-breaking rules yield similar results.

3 As shown in Appendix A, $\bar{X}_{i}$ corresponds to the equilibrium expected expenditure for player $i$. This specification of $\bar{X}_{i}$ allows for a unified treatment of the three possible cases: (a) neither player using all of her available resources, (b) only the weaker player (A) using all of her available resources, and (c) both players $A$ and $B$ using all of their available resources. 
Each contest is modeled as an all-pay auction. The payoff to player $i$ for a bid of $b_{i}^{j}$ in contest $j$ is given by

$$
\pi_{i}^{j}= \begin{cases}v-b_{i}^{j} & \text { if } b_{i}^{j}>b_{-i}^{j} \\ -b_{i}^{j} & \text { if } b_{i}^{j}<b_{-i}^{j}\end{cases}
$$

where ties are handled as described above. Each player's payoff across all $n$ all-pay auctions is the sum of the payoffs across the individual auctions.

The bid provided to each all-pay auction must be nonnegative. For player $i$, the set of feasible bids across the $n$ all-pay auctions is denoted by

$$
\mathfrak{B}_{i}=\left\{\mathbf{b} \in \mathbb{R}_{+}^{n} \mid \sum_{j=1}^{n} b_{i}^{j} \leq X_{i}\right\} .
$$

It will also be useful to define the set of $n$-tuples which exhaust the modified budgets $\bar{X}_{A}$ and $\bar{X}_{B}$. Let $\overline{\mathfrak{B}}_{i}$ denote this set, defined as

$$
\overline{\mathfrak{B}}_{i}=\left\{\mathbf{b} \in \mathbb{R}_{+}^{n} \mid \sum_{j=1}^{n} b_{i}^{j}=\bar{X}_{i}\right\} .
$$

\section{Strategies}

It is well known that there are no pure strategy equilibria for this class of games. A mixed strategy, which we term a distribution of resources, for player $i$ is an $n$-variate distribution function $P_{i}: \mathbb{R}_{+}^{n} \rightarrow[0,1]$ with support (denoted $\operatorname{Supp}\left(P_{i}\right)$ ) contained in the set of player $i$ 's set of feasible bids $\mathfrak{B}_{i}$ and with one-dimensional marginal distribution functions $\left\{F_{i}^{j}\right\}_{j=1}^{n}$, one univariate marginal distribution function for each all-pay auction $j$. The $n$-tuple of player $i$ 's bids across the $n$ all-pay auctions is a random n-tuple drawn from the $n$-variate distribution function $P_{i}$.

The Non-Constant-Sum Colonel Blotto game

The N-C-S Colonel Blotto game, which we label

$$
N C B\left\{X_{A}, X_{B}, n, v\right\}
$$

is the one-shot game in which players compete by simultaneously announcing distributions of resources subject to their budget constraints, each all-pay auction is won by the player that provides the higher bid in that auction (where in the case of a tie the 
tie-breaking rule described above applies), and players' receive the sum of their payoffs across all of the all-pay auctions.

Note that in the non-constant-sum Colonel Blotto game two players simultaneously compete in a set of independent all-pay auctions subject to their respective budget constraints. The presence of the budget constraints gives rise to strategic considerations which are reminiscent of those arising in the single all-pay auction with budgetconstrained bidders (see Che and Gale (1998)). However, in the non-constant-sum Colonel Blotto game the budget constraints hold not within one auction but across the entire set of auctions. As will be seen, the equilibria of these two games differ in fundamental ways.

Before proceeding with the analysis, it is also instructive to compare this formulation with that of the constant-sum Colonel Blotto game. The constant-sum Colonel Blotto game differs from the non-constant-sum game in that in each contest $j$ the payoff to each player $i$ for a bid of $b_{i}^{j}$ is given by

$$
\pi_{i}^{j}= \begin{cases}1 & \text { if } b_{i}^{j}>b_{-i}^{j} \\ 0 & \text { if } b_{i}^{j}<b_{-i}^{j}\end{cases}
$$

where ties are handled as described above. Note that, in the constant-sum game resources which are not allocated to one of the contests have no value; that is, resources are use it or lose it. Each player's payoff across all $n$ contests is the sum of the wins across the contests to which the player provides a higher bid.

\section{Optimal Distributions of Resources}

The following four theorems examine the equilibrium distributions of resources for all symmetric and asymmetric configurations of resource levels. Theorems 1, 2 and 4 characterize the unique sets of equilibrium univariate marginal distributions and the unique equilibrium payoffs. Theorem 3 provides the unique equilibrium payoffs and a pair of equilibrium distributions of resources. ${ }^{4}$

The first two theorems address the portion of the parameter space in which there exists an affine transformation (with respect to the modified budgets) of the equilibrium of the constant-sum game which constitutes an equilibrium of the non-constant-sum game. Once $\left(\bar{X}_{A} / \bar{X}_{B}\right)<(2 / n)$ and $X_{B}>(n-1) X_{A}$ the correspondence between these

${ }^{4}$ In this parameter range there exist a continuum of equilibrium univariate marginal distributions. 
two games breaks down. Theorem 3 is based on the equilibrium of the constant-sum game. However, in this case the transformation entails a more involved modification to the support of the distribution. We conclude with Theorem 4 which constructs entirely new equilibrium distributions of resources in the remaining parameter range.

For the game $N C B\left\{X_{A}, X_{B}, n, v\right\}$, Theorem 1 examines all configurations of resource levels $X_{A}$ and $X_{B}$ which satisfy $(2 / n)<\left(\bar{X}_{A} / \bar{X}_{B}\right) \leq 1$.

Theorem 1 Let $X_{A}, X_{B}, v$, and $n \geq 3$ satisfy $(2 / n) \leq\left(\bar{X}_{A} / \bar{X}_{B}\right) \leq 1$. The pair of $n$ variate distribution functions $P_{A}^{*}$ and $P_{B}^{*}$ constitute a Nash equilibrium of the game $N C B\left\{X_{A}, X_{B}, n, v\right\}$ if and only if they satisfy the two conditions: (1) $\operatorname{Supp}\left(P_{i}^{*}\right) \subset \mathfrak{B}_{i}$ and (2) $P_{i}$ provides the corresponding set of univariate marginal distribution functions $\left\{F_{i}^{j}\right\}_{j=1}^{n}$ outlined below.

For player A the unique set of equilibrium univariate marginal distributions $\left\{F_{A}^{j}\right\}_{j=1}^{n}$ are described as follows

$$
\forall j \in\{1, \ldots, n\} \quad F_{A}^{j}(x)=\left(1-\frac{\bar{X}_{A}}{\bar{X}_{B}}\right)+\frac{x}{(2 / n) \bar{X}_{B}}\left(\bar{X}_{A}\right) \quad \text { for } x \in\left[0, \frac{2}{n} \bar{X}_{B}\right] .
$$

Similarly for player $B$

$$
\forall j \in\{1, \ldots, n\} \quad F_{B}^{j}(x)=\frac{x}{(2 / n) \bar{X}_{B}} \quad \text { for } x \in\left[0, \frac{2}{n} \bar{X}_{B}\right] .
$$

The unique equilibrium expected payoff for player $A$ is $\left(n v \bar{X}_{A} / 2 \bar{X}_{B}\right)-\bar{X}_{A}$, and the unique equilibrium expected payoff for player $B$ is $n v\left(1-\left(\bar{X}_{A} / 2 \bar{X}_{B}\right)\right)-\bar{X}_{B}$.

The existence of a pair of $n$-variate distribution functions which satisfy conditions (1) and (2) of Theorem 1 is provided in Roberson (2006). In particular, Roberson (2006) establishes the existence of $n$-variate distribution functions for which $\operatorname{Supp}\left(P_{i}^{*}\right) \subset \overline{\mathfrak{B}}_{i}$ and that provide the necessary sets of univariate marginal distribution functions given in Theorem 1. The proof of uniqueness of the univariate marginal distribution functions and equilibrium payoffs is given in Appendix A.

An important distinction between the constant-sum and the non-constant-sum versions of the game is that in the constant-sum version each player expends all of her resources with probability one as long as $(1 / n-1) \leq\left(X_{A} / X_{B}\right) \leq 1$. This need not be the case in the non-constant-sum game. In particular there are three possible cases: (a) neither player uses all of her available resources, (b) only (the weaker) player $A$ uses all of her available resources, and (c) both players $A$ and $B$ use all of their available resources.

While it is straightforward to show that any pair of $n$-variate distribution functions which satisfy conditions (1) and (2) of Theorem 1 form an equilibrium, it is useful to 
provide the intuition for this result. We begin with the equilibrium expected payoffs for each player and any $X_{A}$ and $X_{B}$ contained in the portion of the parameter space for which Theorem 1 applies, and then examine these payoffs in each of the three possible cases. Let $P_{B}^{*}$ denote a feasible $n$-variate distribution function for player $B$ with the univariate marginal distributions $\left\{F_{B}^{j}\right\}_{j=1}^{n}$ given in Theroem 1 . If player $B$ is using $P_{B}^{*}$, then player $A$ 's expected payoff $\pi_{A}$, when player $A$ chooses any $n$-tuple of bids $\mathbf{b}_{A} \in \mathfrak{B}_{A}$ (one bid for each of the $n$ all-pay auctions) such that $b_{A}^{j} \in\left[0,(2 / n) \bar{X}_{B}\right]$ for each auction $j$, is

$$
\pi_{A}\left(\mathbf{b}_{A}, P_{B}^{*}\right)=\sum_{j=1}^{n}\left[v F_{B}^{j}\left(b_{A}^{j}\right)-b_{A}^{j}\right] .
$$

Recall that for all $j, F_{B}^{j}(x)=\frac{x}{(2 / n) \bar{X}_{B}}$ for $x \in\left[0,(2 / n) \bar{X}_{B}\right]$. Simplifying yields

$$
\pi_{A}\left(\mathbf{b}_{A}, P_{B}^{*}\right)=\left(\frac{n v}{2 \bar{X}_{B}}-1\right) \sum_{j=1}^{n} b_{A}^{j} .
$$

The expected payoff $\pi_{B}$ to player $B$ from any $n$-tuple of bids across the $n$ all-pay auctions $\mathbf{b}_{B} \in \mathfrak{B}_{B}$ such that $b_{B}^{j} \in\left(0,(2 / n) \bar{X}_{B}\right]$ for each auction $j$ - when player $A$ uses a feasible $n$-variate distribution $P_{A}^{*}$ with the univariate marginal distributions $\left\{F_{A}^{j}\right\}_{j=1}^{n}$ given in Theroem 1 - follows directly,

$$
\pi_{B}\left(\mathbf{b}_{B}, P_{A}^{*}\right)=n v\left(1-\frac{\bar{X}_{A}}{\bar{X}_{B}}\right)+\left(\frac{n v \bar{X}_{A}}{2 \bar{X}_{B}^{2}}-1\right) \sum_{j=1}^{n} b_{B}^{j} .
$$

Observe that neither player can bid below 0 and that bidding above $(2 / n) \bar{X}_{B}$ is suboptimal. Thus, (1) and (2) provide the maximal payoffs (for player $A$ and player $B$ respectively) for any feasible $n$-tuple of bids across the $n$ all-pay auctions.

Suppose that we are in case (a) in which neither player uses all of her available resources. Case (a) corresponds to the situation in which the total value of the $n$ auctions $n v$ is low enough relative to the players' budgets that neither player has incentive to commit all of her resources. If player $A$ does not use all of her budget, then from $\bar{X}_{A}=\min \left\{X_{A}, n v / 2\right\}$ it must be that $X_{A}>(n v / 2)$ and so $\bar{X}_{A}=(n v / 2)$. Similarly from $\bar{X}_{B}=\min \left\{X_{B}, \sqrt{n v \bar{X}_{A} / 2}\right\}$, it follows that if player $A$ (the weaker player) is not using all of her budget then $\bar{X}_{B}=(n v / 2)$. Given that $\bar{X}_{A}=\bar{X}_{B}=(n v / 2)$, the expected payoffs given in (1) and (2) are $\pi_{A}\left(\mathbf{b}_{A}, P_{B}^{*}\right)=0$ and $\pi_{B}\left(\mathbf{b}_{B}, P_{A}^{*}\right)=0$ respectively. Observe that in case (a) neither player has incentive to change the aggregate level of resources that they commit to the $n$ all-pay auctions. That is, given that the opponent is using the equilibrium strategy, the expected payoff to each player is independent of the aggregate level of resources that they commit across the $n$ all-pay auctions. 
Now suppose that we are in case (b) in which only player $A$ uses all of her budget. Case (b) corresponds to the situation in which the total value of the $n$ all-pay auctions $n v$ is high enough that the weaker player optimally commits all of her resources but not so high that the stronger player must also commit all of her resources to the $n$ all-pay auctions. From the proceeding discussion it follows that $X_{A} \leq(n v / 2)$ and thus $\bar{X}_{A}=X_{A}$. If player $B$ is not using all of her budget then from $\bar{X}_{B}=\min \left\{X_{B}, \sqrt{n v X_{A} / 2}\right\}$, it must be that $X_{B}>\sqrt{n v X_{A} / 2}$ and so $\bar{X}_{B}=\sqrt{n v X_{A} / 2}$. Inserting $\bar{X}_{A}$ and $\bar{X}_{B}$ into (1) and (2) and simplifying yields

$$
\pi_{A}\left(\mathbf{b}_{A}, P_{B}^{*}\right)=\left(\sqrt{\frac{n v}{2 X_{A}}}-1\right) \sum_{j=1}^{n} b_{A}^{j}
$$

and

$$
\pi_{B}\left(\mathbf{b}_{B}, P_{A}^{*}\right)=n v\left(1-\sqrt{\frac{2 X_{A}}{n v}}\right) .
$$

Recall that in case (b) $X_{A} \leq(n v / 2)$ and so $\left(\sqrt{n v / 2 X_{A}}-1\right) \geq 0$. From (3) we see that player $A$ is indifferent with regards to which all-pay auctions to commit resources to, but has incentive to increase her aggregate level of resource commitment across the $n$ all-pay auctions. However in case (b), player $A$ is at her budget constraint and her equilibrium distribution of resources $P_{A}^{*}$ expends her budget with probability one. ${ }^{5}$ From (4) we see that the expected payoff to player $B$ is independent of the aggregate level of resources that she commits across the $n$ all-pay auctions (so long as she commits a strictly positive level of resources to each auction), and so player $B$ does not have incentive to change the aggregate level of resources that she commits to the $n$ all-pay auctions.

Finally, suppose that we are in case (c) in which both players use all of their budgets. Case (c) corresponds to the situation in which the total value of the $n$ all-pay auctions $n v$ is high enough that both players optimally commit all of their resources to the $n$ all-pay auctions. Thus, $\bar{X}_{A}=X_{A}$ and $\bar{X}_{B}=X_{B}$. From (1) and (2) it follows that

$$
\pi_{A}\left(\mathbf{b}_{A}, P_{B}^{*}\right)=\left(\frac{n v}{2 X_{B}}-1\right) \sum_{j=1}^{n} b_{A}^{j}
$$

and

$$
\pi_{B}\left(\mathbf{b}_{B}, P_{A}^{*}\right)=n v\left(1-\frac{X_{A}}{X_{B}}\right)+\left(\frac{n v X_{A}}{2 X_{B}^{2}}-1\right) \sum_{j=1}^{n} b_{B}^{j} .
$$

5 Recall that Roberson (2006) establishes the existence of $n$-variate distribution functions for which $\operatorname{Supp}\left(P_{i}^{*}\right) \subset \overline{\mathfrak{B}}_{i}$, and that in this case $\bar{X}_{A}=X_{A}$. It follows directly that player A expends her budget with probability one. 
In case (c), $X_{A}<(n v / 2)$ and $X_{B}<\sqrt{n v X_{A} / 2}<(n v / 2)$. Observe in (5) that $\left(\left(n v / 2 X_{B}\right)-\right.$ 1) $>0$ and, thus, player $A$ has incentive to increase her aggregate level of resource commitment across the $n$ all-pay auctions, but in her equilibrium distribution of resources $P_{A}^{*}$ she is already at her budget constraint with probability one. Similarly, in (6) $\left(\left(n v X_{A} / 2 X_{B}^{2}\right)-1\right)>0$ and, thus, player $B$ has incentive to increase her aggregate level of resource commitment across the $n$ all-pay auctions, but in her equilibrium distribution of resources $P_{B}^{*}$ she is already at her budget constraint with probability one.

Given that Roberson (2006) demonstrates the existence of a pair of $n$-variate distributions that satisfy conditions (1) and (2) of Theorem 1, it follows from the arguments given above that such a pair of $n$-variate distribution functions constitute an equilibrium in all three cases (a), (b), and (c). The proof of the uniqueness of the sets of univariate marginal distributions is given in Appendix A.

The following Theorem addresses the remaining portion of the parameter space for which there exists an affine transformation of the equilibrium of the constant-sum game which constitutes an equilibrium of the non-constant-sum game.

Theorem 2 Let $X_{A}, X_{B}, v$, and $n \geq 3$ satisfy $\left(\bar{X}_{A} / \bar{X}_{B}\right)<(2 / n)$ and $X_{B} \leq(n-1) X_{A}$. The pair of $n$-variate distribution functions $P_{A}^{*}$ and $P_{B}^{*}$ constitute a Nash equilibrium of the game $N C B\left\{X_{A}, X_{B}, n, v\right\}$ if and only if they satisfy the two conditions: (1) $\operatorname{Supp}\left(P_{i}^{*}\right) \subset \mathfrak{B}_{i}$ and (2) $P_{i}$ provides the corresponding set of univariate marginal distribution functions $\left\{F_{i}^{j}\right\}_{j=1}^{n}$ outlined below.

For player A the unique set of equilibrium univariate marginal distribution functions $\left\{F_{A}^{j}\right\}_{j=1}^{n}$ are described as follows

$$
\forall j \in\{1, \ldots, n\} \quad F_{A}^{j}(x)=\left(1-\frac{2}{n}\right)+\frac{x}{X_{A}}\left(\frac{2}{n}\right) \quad \text { for } x \in\left[0, X_{A}\right] .
$$

Similarly for player $B$

$$
\forall j \in\{1, \ldots, n\} \quad F_{B}^{j}(x)=\left\{\begin{array}{ll}
\frac{2 x\left(X_{A}-\frac{X_{B}}{n}\right)}{\left(X_{A}\right)^{2}} & \text { for } x \in\left[0, X_{A}\right) . \\
1 & \text { for } x \geq X_{A}
\end{array} .\right.
$$

The unique equilibrium expected payoff for player $A$ is $n v\left((2 / n)-\left(\left(2 X_{B}\right) /\left(n^{2} X_{A}\right)\right)\right)-$ $X_{A}$, and the unique equilibrium expected payoff for player $B$ is $n v(1-(2 / n))+$ $n v\left(\left(2 X_{B}\right) /\left(n^{2} X_{A}\right)\right)-X_{B}$.

The existence of a pair of $n$-variate distribution functions which satisfy conditions (1) and (2) of Theorem 2 is provided in Roberson (2006). The proof of uniqueness of the univariate marginal distributions and equilibrium payoffs is given in Appendix A. 
Before proceeding with a sketch of the proof that a pair of $n$-variate distributions that satisfy conditions (1) and (2) of Theorem 2 form an equilibrium, it is helpful to trace out the Theorem 2 parameter range. Since $\bar{X}_{B}=\min \left\{X_{B}, \sqrt{n v \bar{X}_{A} / 2}\right\}$ and $\left(\bar{X}_{A} / \bar{X}_{B}\right)<(2 / n)$ it follows that

$$
\bar{X}_{A}<\frac{2}{n} \bar{X}_{B} \leq \sqrt{\frac{2 v \bar{X}_{A}}{n}}
$$

and so $\bar{X}_{A}<(2 v / n)$. Therefore it must be the case that $\bar{X}_{A}=X_{A}$. It also follows that $X_{B} \leq(n-1) X_{A}$ combined with $(n-1) X_{A}<\sqrt{n v X_{A} / 2}$ implies that $\bar{X}_{B}=X_{B}$. Thus, the Theorem 2 parameter range is given by $0 \leq X_{A}<(2 v / n)$ and $(n / 2) X_{A}<X_{B} \leq(n-1) X_{A}$

Returning to the sketch of the proof that a pair of $n$-variate distributions that satisfy conditions (1) and (2) of Theorem 2 form an equilibrium, let $P_{B}^{*}$ denote a feasible $n$ variate distribution for player $B$ with the univariate marginal distributions $\left\{F_{B}^{j}\right\}_{j=1}^{n}$ given in Theorem 2. If player $B$ is using $P_{B}^{*}$, then player $A$ 's expected payoff $\pi_{A}$, when player $A$ chooses any $n$-tuple of bids $\mathbf{b}_{A} \in \mathfrak{B}_{A}$ such that $b_{A}^{j} \in\left[0, X_{A}\right)$ for each auction $j$, is

$$
\pi_{A}\left(\mathbf{b}_{A}, P_{B}^{*}\right)=\left(\frac{2 v\left(X_{A}-\left(X_{B} / n\right)\right)}{X_{A}^{2}}-1\right) \sum_{j=1}^{n} b_{A}^{j} .
$$

Note that $\left(2 v / X_{A}^{2}\right)\left(X_{A}-\left(X_{B} / n\right)\right)-1 \geq 0$ is equivalent to $X_{B} \leq\left(n-\left(n X_{A} / 2 v\right)\right) X_{A}$. Since $X_{A}<(2 v / n)$, it follows from (7) that player $A$ has incentive to expend all of her available resources in the $n$ all-pay auctions not only in expectation but with certainty.

Similarly, the expected payoff $\pi_{B}$ to player $B$ from any $n$-tuple of bids across the $n$ all-pay auctions $\mathbf{b}_{B} \in \mathfrak{B}_{B}$ such that $b_{B}^{j} \in\left(0, X_{A}\right]$ for each auction $j$, when player $A$ uses a feasible $n$-variate distribution $P_{A}^{*}$ with the univariate marginal distributions $\left\{F_{A}^{j}\right\}_{j=1}^{n}$ given in Theroem 2, is

$$
\pi_{B}\left(\mathbf{b}_{B}, P_{A}^{*}\right)=n v\left(1-\frac{2}{n}\right)+\left(\frac{2 v}{n X_{A}}-1\right) \sum_{j=1}^{n} b_{B}^{j} .
$$

Since $X_{A}<(2 v / n)$ it follows that $\left(2 v / n X_{A}\right)-1>0$, and, thus, player $B$ has incentive to expend all of her available resources in the $n$ all-pay auctions with certainty.

Given that Roberson (2006) demonstrates the existence of a pair of $n$-variate distributions that result in the sets of univariate marginal distributions given in Theorem 2 and that satisfy the budget restriction with probability 1, it follows from the arguments given above that such a pair of $n$-variate distribution functions constitute an equilibrium. The proof of uniqueness of the univariate marginal distributions is given in Appendix A. 
While the first two theorems involve affine transformations (with respect to the modified budgets) of the equilibrium of the constant-sum game, once $\left(\bar{X}_{A} / \bar{X}_{B}\right)<(2 / n)$ and $X_{B}>(n-1) X_{A}$ the correspondence between the constant-sum and non-constantsum games breaks down. For the remaining parameter range, Theorems 3 and 4 construct new equilibrium joint distributions. Theorem 3, which addresses the case that $\left(\bar{X}_{A} / \bar{X}_{B}\right)>(2 / n)$ and $\min \left\{n X_{A},(n-2) X_{A}+(2 v / n)\right\}>X_{B}>(n-1) X_{A}$, is also based on the equilibrium of the constant-sum game but includes a more involved modification of the support. Theorem 4, which addresses the remaining case that $\left(\bar{X}_{A} / \bar{X}_{B}\right)<(2 / n)$ and $X_{B} \geq \min \left\{n X_{A},(n-2) X_{A}+(2 v / n)\right\}$ (note that if $X_{A}<(2 v / n)$ then $\min \left\{n X_{A},(n-\right.$ 2) $\left.\left.X_{A}+(2 v / n)\right\}>(n-1) X_{A}\right)$, constructs entirely new equilibrium distributions of resources.

Before turning to the statements of Theorems 3 and 4, observe that while the relationship between the constant-sum and and non-constant-sum versions of the game is linear with respect to the modified budgets - as long as the level of asymmetry between the players' budgets is below the threshold given in Theorem 2 - the relationship between these games with respect to the aggregate resource levels is highly non-linear. Panel (i) of Figure 1 illustrates the regions of the parameter space corresponding to each of the four theorems in the non-constant-sum game, and Panel (ii) of Figure 1 illustrates the regions which correspond, for the constant-sum game, to Theorems 2, 3, and 5 of Roberson (2006).

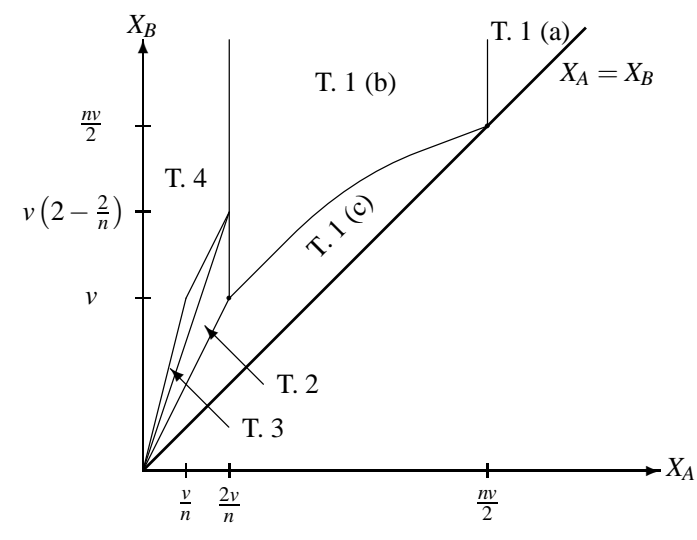

(i) Non-constant-sum

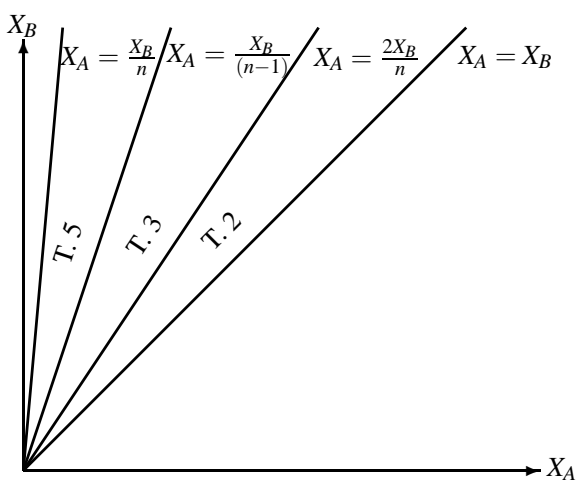

(ii) Constant-Sum

Fig. 1 Resource Level Configurations 
In the constant-sum game, four rays emanating from the origin partition the parameter space into four disjoint regions. As shown in Panel (ii) of Figure 1, these regions are delineated by (1) $X_{A}=\left(X_{B} /(n-1)\right)$, (2) $X_{A}=\left(2 X_{B} / n\right)$, and (3) $X_{A}=X_{B}$. While Theorems 1, 2 and 3 of this paper (the non-constant-sum game) are transformations of Theorems 2, 3 and 5 of Roberson (2006) (the constant-sum game) respectively, the corresponding parameter regions differ in nontrivial ways. The complicating factor in the relationship between the two versions of the game is the strategic considerations arising from the use it or lose it feature of the constant-sum formulation and the corresponding relaxation of this feature in the non-constant-sum formulation. In particular, recall that in the non-constant-sum game with resource levels which satisfy $(2 / n) \leq\left(\bar{X}_{A} / \bar{X}_{B}\right) \leq 1$ (as in Theorem 1) there were three possible cases: (a) neither player uses all of their available resources, (b) only (the weaker) player $A$ uses all of her available resources, and (c) both players $A$ and $B$ use all of their available resources. (The regions corresponding to each of these cases is labeled in panel (i) of Figure 1.)

Furthermore, in the region in which $X_{A}<\left(X_{B} / n\right)$ the constant-sum game is trivial since resources are use it or lose it and the stronger player (B) has a sufficient level of resources to win each of the $n$ contests with certainty. In this is region there is no relationship between the two games. Due to the relaxation of the use it or lose it feature, the non-constant-sum game never becomes trivial, and for the non-constant-sum game Theorem 4 constructs entirely new equilibrium distributions of resources in the remaining parameter range.

In the case that $\left(\bar{X}_{A} / \bar{X}_{B}\right)<(2 / n)$ and $\min \left\{n X_{A},(n-2) X_{A}+(2 v / n)\right\}>X_{B}>(n-$ 1) $X_{A}$ Theorem 2 would provide the unique set of equilibrium marginal distributions if a sufficient $n$-variate distribution function were to exist for each player. As in the corresponding constant-sum parameter range, such a joint distribution fails to exist for player B. One equilibrium is given by an extension of the case of $n=2$ with asymmetric forces discussed by Gross and Wagner (1950). The set of equilibrium univariate marginal distributions is not unique, but the equilibrium payoffs are unique.

Theorem 3 Define $k=\left\lceil\left(X_{A}\right) /\left(X_{B}-X_{A}(n-1)\right)\right\rceil$. Let $X_{A}, X_{B}, v$, and $n \geq 3$ satisfy $\left(\bar{X}_{A} / \bar{X}_{B}\right)<(2 / n)$ and $\min \left\{n X_{A},(n-2) X_{A}+(2 v / n)\right\}>X_{B}>(n-1) X_{A}$. A Nash equilibrium of the game $N C B\left\{X_{A}, X_{B}, v, n\right\}$ is for each player to allocate her resources according to the following $n$-variate distributions:

Player A randomly allocates 0 resources to $n-2$ of the all-pay auctions, each all-pay auction chosen with equal probability, $(n-2) / n$. On the remaining 2 all-pay auctions player A utilizes a bivariate distribution function with $k$ mass points, each mass point 
receiving the same weight, $\left(1-\left(n X_{A}\right) /(2 v)\right) / k$. Player A's mass points on these two remaining all-pay auctions are located at the points

$$
\left((k-1-i) \frac{X_{A}}{k-1}, i \frac{X_{A}}{k-1}\right), i=0, \ldots, k-1 .
$$

Player A uniformly distributes the remaining $\left(n X_{A}\right) /(2 v)$ of the mass along her budget line $\left\{\left(x_{1}, x_{2}\right) \mid x_{1}+x_{2}=X_{A}\right\}$.

Player $B$ randomly allocates $X_{A}$ forces to $n-2$ all-pay auctions, each all-pay auction chosen with equal probability, $(n-2) / n$. On the remaining 2 all-pay auctions player $B$ utilizes a bivariate distribution function with $k$ mass points, each mass point receiving the same weight, $\left(1-n\left(X_{B}-X_{A}(n-2)\right) /(2 v)\right) /(k)$. Player B's mass points on the 2 remaining battlefields are located at

$$
\left(X_{A}-i \frac{n X_{A}-X_{B}}{k-1}, X_{A}-(k-1-i) \frac{n X_{A}-X_{B}}{k-1}\right), i=0, \ldots, k-1 .
$$

Player $B$ uniformly distributes the remaining $\left(X_{B}-X_{A}(n-2)\right) /(2 v)$ of the mass along her budget line $\left\{\left(x_{1}, x_{2}\right) \mid x_{1}+x_{2}=X_{B}-X_{A}(n-2)\right\}$ and the two line segments $\left\{\left(x_{1}, x_{2}\right) \mid x_{1}=X_{A}\right.$ and $\left.0 \leq x_{2} \leq X_{B}-X_{A}(n-1)\right\}$, and $\left\{\left(x_{1}, x_{2}\right) \mid x_{2}=X_{A}\right.$ and $0 \leq x_{1} \leq$ $\left.X_{B}-X_{A}(n-1)\right\}$.

The unique equilibrium expected payoff for player $A$ is $v(k-1)\left((2 / n)-\left(\left(X_{B}-\right.\right.\right.$ $\left.\left.\left(X_{A}(n-2)\right) / v\right)\right) / k$, and the unique equilibrium expected payoff for player $B$ is $(v-$ $\left.X_{A}\right)(n-2)+((v 2(n-2)) / n)+v(k-1)\left((2 / n)-\left(X_{A} / v\right)\right) / k$.

The proof of Theorem 3 is given in Appendix B.

The following Theorem constructs entirely new equilibrium distributions of resources for the portion of the parameter space in which the correspondence between the constantsum and non-constant-sum versions of the game breaks down.

Theorem 4 Let $X_{A}, X_{B}, v$, and $n \geq 3$ satisfy $\left(\bar{X}_{A} / \bar{X}_{B}\right)<(2 / n)$ and $X_{B} \geq \min \left\{n X_{A},(n-\right.$ 2) $\left.X_{A}+(2 v / n)\right\}$. The pair of $n$-variate distribution functions $P_{A}^{*}$ and $P_{B}^{*}$ constitute a Nash equilibrium of the game $N C B\left\{X_{A}, X_{B}, n, v\right\}$ if and only if they satisfy the two conditions: (1) $\operatorname{Supp}\left(P_{i}^{*}\right) \subset \mathfrak{B}_{i}$ and (2) $P_{i}$ provides the corresponding set of univariate marginal distribution functions $\left\{F_{i}^{j}\right\}_{j=1}^{n}$ outlined below.

For player A the unique set of equilibrium univariate marginal distribution functions $\left\{F_{A}^{j}\right\}_{j=1}^{n}$ are described as follows

$$
\forall j \in\{1, \ldots, n\} \quad F_{A}^{j}(x)=\left(1-\frac{X_{A}}{v}\right)+\frac{x}{v} \quad \text { for } x \in\left[0, X_{A}\right] .
$$


Similarly for player $B$

$$
\forall j \in\{1, \ldots, n\} \quad F_{B}^{j}(x)=\left\{\begin{array}{ll}
\frac{x}{v} & \text { for } x \in\left[0, X_{A}\right) \\
1 & \text { for } x \geq X_{A}
\end{array} .\right.
$$

The unique equilibrium expected payoff for player $A$ is 0 , and the unique equilibrium expected payoff for player $B$ is $n v\left(1-\left(X_{A} / v\right)\right)$.

The existence of $n$-variate distributions which satisfy conditions (1) and (2) of Theorem 4 is provided in Appendix C. The proof of uniqueness of the univariate marginal distributions and equilibrium payoffs is given in Appendix A.

To see that these two sets of univariate marginal distributions form an equilibrium in the Theorem 4 parameter region, let $P_{B}^{*}$ denote a feasible $n$-variate distribution for player $B$ with the univariate marginal distributions $\left\{F_{B}^{j}\right\}_{j=1}^{n}$ given in Theorem 4. If player $B$ is using $P_{B}^{*}$, then player $A$ 's expected payoff $\pi_{A}$, when player $A$ chooses any $n$-tuple of bids $\mathbf{b}_{A} \in \mathfrak{B}_{A}$ is

$$
\pi_{A}\left(\mathbf{b}_{A}, P_{B}^{*}\right)=0 .
$$

From (9), player $A$ does not have incentive to increase or decrease her level of resource commitment in the $n$ all-pay auctions.

Similarly, the expected payoff $\pi_{B}$ to player $B$ from any $n$-tuple of bids across the $n$ all-pay auctions $\mathbf{b}_{B} \in \mathfrak{B}_{B}$ such that $b_{B}^{j} \in\left(0, X_{A}\right]$ for each auction $j$, when player $A$ uses a feasible $n$-variate distribution $P_{A}^{*}$ with the univariate marginal distributions $\left\{F_{A}^{j}\right\}_{j=1}^{n}$ given in Theroem 4, is

$$
\pi_{B}\left(\mathbf{b}_{B}, P_{A}^{*}\right)=n v\left(1-\frac{X_{A}}{v}\right) .
$$

Thus, player $B$ also has no incentive to increase or decrease her level of resource commitment in the $n$ all-pay auctions.

Given that Appendix C provides the construction of $n$-variate distribution functions which satisfy conditions (1) and (2) of Theorem 4, it follows from the arguments given above that such a pair of $n$-variate distribution functions constitute an equilibrium. The proof of uniqueness of the univariate marginal distributions is given in Appendix A.

\section{Conclusion}

Kvasov (2007) introduces a non-constant-sum version of the Colonel Blotto game which relaxes the "use it or lose it" feature of the traditional constant-sum formulation of the game. In the case of symmetric budgets, that paper establishes that a suitable affine 
transformation of the constant-sum equilibrium is an equilibrium of the non-constantsum game. In this paper we characterize all asymmetric parameter configurations of the non-constant-sum version of the Colonel Blotto game. As long as the player's budgets are not too asymmetric, a suitable affine transformation (with respect to the modified budgets) of the constant-sum asymmetric equilibrium (Roberson 2006) is an equilibrium of the non-constant-sum asymmetric game. However, once the players' budgets are sufficiently asymmetric this correspondence breaks down. In this parameter range, we construct entirely new equilibrium joint distributions.

\section{Appendix A}

This appendix characterizes the sets of equilibrium univariate marginal distributions in Theorems 1, 2, and 4. Given that the non-constant-sum Colonel Blotto game is a set of independent and simultaneous all-pay auctions with (symmetric and asymmetric) budget constraints, the characterization of the equilibrium univariate marginal distributions follows along the line of argument for the characterization of the all-pay auction by Hillman and Riley (1989) and Baye, Kovenock and de Vries (1996). Roberson (2006) establishes the existence of feasible $n$-variate distribution functions for Theorems 1 and 2. The existence of such $n$-variate distribution functions for Theorem 4 is given in Appendix $\mathrm{C}$.

In the discussion that follows we will focus on Theorem 1. The proofs for Theorems 2 and 4 follow directly. Let $\bar{s}_{i}^{j}$ and $\underline{s}_{i}^{j}$ denote the upper and lower bounds of player $i$ 's distribution of resources for all-pay auction $j$.

Recall that in Theorem 1 the corresponding parameter space is $(2 / n) \leq\left(\bar{X}_{A} / \bar{X}_{B}\right) \leq 1$. It will also be convenient to note that, for a given $P_{-i}$, with the set of univariate marginal distribution functions $\left\{F_{-i}^{j}\right\}_{j=1}^{n}$, the Lagrangian of each player $i$ 's optimization problem 6 can be written as

$$
\max _{\left\{F_{i}^{j}\right\}_{j=1}^{n}}\left(1+\lambda_{i}\right) \sum_{j=1}^{n}\left[\int_{0}^{\infty}\left[\frac{v}{\left(1+\lambda_{i}\right)} F_{-i}^{j}(x)-x\right] d F_{i}^{j}\right]+\lambda_{i} X_{i}
$$

where the set of univariate marginal distribution functions $\left\{F_{i}^{j}\right\}_{j=1}^{n}$ satisfy the constraint that there exists a mapping of the set of univariate marginal distributions into 6 This formulation assumes that for all battlefields the players' univariate marginal distributions do not place an atom on the same value. However, it is straightforward to incorporate the tie-breaking rule into the Lagrangian of each player's optimization problem. 
a joint distribution (an $n$-copula), $C$, such that the support of the $n$-variate distribution $C\left(F_{i}^{1}\left(x^{1}\right), \ldots, F_{i}^{n}\left(x^{n}\right)\right)$ is contained in $\mathfrak{B}_{i}$.

The first two lemmas follow along the lines of the proofs for the symmetric case given in Kvasov (2007). ${ }^{7}$

Lemma 1 In any equilibrium $\left\{F_{i}^{j}, F_{-i}^{j}\right\}_{j \in\{1, \ldots, n\}}$, no $F_{i}^{j}$ can place an atom in the half open interval $\left(0, \bar{s}^{j}\right]$.

Lemma 2 For each $j \in\{1, \ldots, n\}$ and for each $i \in\{A, B\}, \frac{v}{1+\lambda_{i}} F_{-i}^{j}(x)-x$ is constant $\forall$ $x \in\left(0, \bar{s}^{j}\right]$.

The next two lemmas follow along the lines of the proofs in Baye, Kovenock, and de Vries (1996).

Lemma 3 For each $j \in\{1, \ldots, n\}, \bar{s}_{-i}^{j}=\bar{s}_{i}^{j}=\bar{s}^{j}$.

Lemma $4 \forall j \in\{1, \ldots, n\}, F_{B}^{j}(0)=0$ and, thus, $\frac{v}{1+\lambda_{A}} F_{B}^{j}(x)-x=0 \forall x \in\left[0, \bar{s}^{j}\right]$.

The following lemma characterizes the relationship between $\lambda_{A}$ and $\lambda_{B}$. Let $\bar{X}_{i}$ denote player $i$ 's expected expenditure, that is

$$
\bar{X}_{i}=\sum_{j=1}^{n} \int_{0}^{\bar{s}^{j}} x d F_{i}^{j}(x) .
$$

Lemma 5 In equilibrium $\left(1+\lambda_{A}\right)=\left(1+\lambda_{B}\right) \frac{\bar{X}_{B}}{\bar{X}_{A}}$.

Proof From Lemma 2, it follows that $d F_{A}^{j}(x)=\frac{\left(1+\lambda_{B}\right)}{v} d x$ and $d F_{B}^{j}(x)=\frac{\left(1+\lambda_{A}\right)}{v} d x$ for all $x \in\left[0, \bar{s}^{j}\right]$. Substituting these expressions into equation (12), we have $\left(1+\lambda_{A}\right)=$ $\bar{X}_{B} \frac{2 v}{\sum_{j}\left(\bar{s}^{j}\right)^{2}}$ and $\left(1+\lambda_{B}\right)=\bar{X}_{A} \frac{2 v}{\sum_{j}\left(\bar{s}^{j}\right)^{2}}$. The result follows directly.

The following lemma establishes the value of $\bar{s}^{j}$.

7 While the characterization of the equilibrium univariate marginal distributions for the constant-sum and non-constant-sum versions of the game follow along similar lines, there are important distinctions. In both cases, Lemmas 1-4 are established using feasible points in the support. In the non-constant-sum game Kvasov (2007) uses a separating hyperplane argument to prove that each of the univariate marginal distributions is strictly increasing and continuous on its support. Conversely, in the constant-sum game Roberson (2006) relies on properties of two-player constant-sum games (namely, interchangeability of equilibrium strategies and uniqueness of equilibrium payoffs) to establish these properties of the univariate marginal distributions. 
Lemma $6 \bar{s}^{j}=\frac{v}{1+\lambda_{A}}$.

Proof From Lemma 2, we know that for each player $i$ and any battlefield $j, \frac{v}{1+\lambda_{i}} F_{-i}^{j}(x)-$ $x$ is constant $\forall x \in\left(0, \bar{s}^{j}\right]$. It then follows that player $i$ would never use a strategy that provides offers in $\left(\frac{v}{1+\lambda_{i}}, \infty\right)$ since an offer of zero strictly dominates such a strategy. It follows from Lemma 4 that $\frac{v}{1+\lambda_{A}} \leq \frac{v}{1+\lambda_{B}}$. Thus $\bar{s}^{j} \leq \frac{v}{1+\lambda_{A}}$ and $\forall x \in\left(0, \bar{s}^{j}\right]$

$$
\frac{v}{1+\lambda_{i}} F_{-i}^{j}(x)-x \geq \frac{v}{1+\lambda_{i}}-\bar{s}^{j} .
$$

By way of contradiction, assume that $\bar{s}^{j}<\frac{v}{1+\lambda_{A}}$. By allocating a level of force to battlefield $j$ that is greater than $\bar{s}^{j}$ by an arbitrarily small amount, player $A$ can earn arbitrarily close to $\frac{v}{1+\lambda_{A}}-\bar{s}^{j}>0$ on battlefield $j$, which contradicts Lemma 4.

The following lemma establishes that there exists a unique pair $\lambda_{A}, \lambda_{B}$ that satisfies the budget constraint.

Lemma 7 There exists a unique value for $\lambda_{A}$, and thus for $\lambda_{B} \cdot \lambda_{A}=\frac{n v}{2 \bar{X}_{B}}-1$ and thus $\lambda_{B}=\frac{n v \bar{X}_{A}}{2 \bar{X}_{B}^{2}}-1$.

Proof The expected expenditure determines the unique pair $\lambda_{A}, \lambda_{B}$. Thus, $\lambda_{A}$ solves

$$
\frac{n\left(1+\lambda_{A}\right)}{v} \int_{0}^{\frac{v}{1+\lambda_{A}}} x \lambda_{A} d x=\bar{X}_{B}
$$

Solving for $\lambda_{A}$ we have that

$$
\lambda_{A}=\frac{n v}{2 \bar{X}_{B}}-1
$$

It follows directly from Lemma 5 that

$$
\lambda_{B}=\frac{n v \bar{X}_{A}}{2 \bar{X}_{B}^{2}}-1
$$

To complete the proof of Lemma 7, recall the three possible cases: (a) neither player uses all of her available resources, (b) only (the weaker) player $A$ uses all of her available resources, and (c) both players $A$ and $B$ use all of their available resources.

In case (a) $\lambda_{A}=\lambda_{B}=0$. From (13) and (14) we have that $\bar{X}_{B}=\frac{n v}{2}$ and $\bar{X}_{B}=\sqrt{\frac{n v \bar{X}_{A}}{2}}$. Thus, $X_{B} \geq \frac{n v}{2}$ and $X_{A} \geq \frac{n v}{2}$. In case (b) $\lambda_{A}>0$ and $\lambda_{B}=0$. From (13) and (14) we have that $\bar{X}_{B}<\frac{n v}{2}$ and $\bar{X}_{B}=\sqrt{\frac{n v \bar{X}_{A}}{2}}$. Thus, $X_{B} \geq \sqrt{\frac{n v X_{A}}{2}}$ and $X_{A}<\frac{n v}{2}$. In case (c) $\lambda_{A}>0$ and $\lambda_{B}>0$. From (13) and (14) we have that $\bar{X}_{B}<\frac{n v}{2}$ and $\bar{X}_{B}<\sqrt{\frac{n v \bar{X}_{A}}{2}}$. Thus, $X_{B}<\sqrt{\frac{n v X_{A}}{2}}$ and $X_{A}<\frac{n v}{2}$.

To summarize $\bar{X}_{B}=\min \left\{X_{B}, \sqrt{\frac{n v \bar{X}_{A}}{2}}\right\}$ and $\bar{X}_{A}=\min \left\{X_{A}, \frac{n v}{2}\right\}$. Thus, for any pair $X_{A}$, $X_{B}$ there exists a unique pair $\bar{X}_{A}, \bar{X}_{B}$, and a unique pair $\lambda_{A}, \lambda_{B}$. 
This completes the characterization of the sets of equilibrium univariate marginal distributions in Theorem 1. The proofs for Theorems 2 and 4 follow along similar lines.

\section{Appendix B}

The proof of Theorem 3, stated below, establishes the existence of an equilibrium in the game $N C B\left\{X_{A}, X_{B}, n\right\}$ for $X_{A}, X_{B}$, and $n \geq 3$ such that $\frac{\bar{X}_{A}}{\bar{X}_{B}}<\frac{2}{n}$ and $\min \left\{n X_{A},(n-2) X_{A}+\right.$ $\left.\frac{2 v}{n}\right\}>X_{B}>(n-1) X_{A}$. The proof of uniqueness of the equilibrium payoffs follows directly. In the discussion that follows, recall that $k=\left\lceil\frac{X_{A}}{X_{B}-X_{A}(n-1)}\right\rceil$, and thus, $2 \leq k<\infty$.

First, the strategies in the statement of Theorem 5 are feasible since for player $A$

$$
(k-1-i) \frac{X_{A}}{k-1}+i \frac{X_{A}}{k-1}=X_{A},
$$

and for player $B$

$$
X_{A}(n-2)+X_{A}-i \frac{n X_{A}-X_{B}}{k-1}+X_{A}-(k-1-i) \frac{n X_{A}-X_{B}}{k-1}=X_{B}
$$

for all $i=0, \ldots, k-1$.

Second, each player is indifferent between each point in the support of their strategy. For this equilibrium the univariate marginal distributions for player $A$ and $\forall j \in$ $\{1, \ldots, n\}$ are

$$
F_{A}^{j}(x)= \begin{cases}\frac{n-2}{n}+\frac{\left(\frac{2}{n}-\frac{X_{A}}{v}\right)}{k}+\frac{x}{v} & \text { if } x \in\left[0, \frac{X_{A}}{k-1}\right) \\ \frac{n-2}{n}+\frac{2\left(\frac{2}{n}-\frac{X_{A}}{v}\right)}{k}+\frac{x}{v} & \text { if } x \in\left[\frac{X_{A}}{k-1}, \frac{2 X_{A}}{k-1}\right) \\ \vdots & \vdots \\ \frac{n-2}{n}+\frac{(i+1)\left(\frac{2}{n}-\frac{X_{A}}{v}\right)}{k}+\frac{x}{v} & \text { if } x \in\left[\frac{i X_{A}}{k-1}, \frac{(i+1) X_{A}}{k-1}\right) \\ \vdots & \vdots \\ \frac{n-2}{n}+\frac{(k-1)\left(\frac{2}{n}-\frac{X_{A}}{v}\right)}{k}+\frac{x}{v} & \text { if } x \in\left[\frac{(k-2) X_{A}}{k-1}, X_{A}\right) \\ 1 & \text { if } x \geq X_{A}\end{cases}
$$


Similarly for player $B$ and $\forall j \in\{1, \ldots, n\}$ we have

$$
F_{B}^{j}(x)= \begin{cases}\frac{x}{v} & \text { if } x \in\left[0, X_{B}-X_{A}(n-1)\right) \\ \frac{\left(\frac{2}{n}-\frac{X_{B}-X_{A}(n-2)}{v}\right)}{k}+\frac{x}{v} & \text { if } x \in\left[X_{B}-X_{A}(n-1), X_{A}-\frac{(k-2)\left(n X_{A}-X_{B}\right)}{k-1}\right) \\ \vdots & \vdots \\ \vdots & \text { if } x \in\left[X_{A}-\frac{(k-1-i)\left(n X_{A}-X_{B}\right)}{k-1}, X_{A}-\frac{(k-2-i)\left(n X_{A}-X_{B}\right)}{k-1}\right) . \\ \frac{(k-1)\left(\frac{2}{n}-\frac{X_{B}-X_{A}(n-2)}{v}\right)}{k}+\frac{x}{v} & \text { if } x \in\left[X_{A}-\frac{n X_{A}-X_{B}}{k-1}, X_{A}\right) \\ 1 & \text { if } x \geq X_{A}\end{cases}
$$

We begin with player $A$ 's expected payoff for each of her $k$ mass points, and then examine the remaining uniform randomization. Note that for $i=1, \ldots, k-1,{ }^{8}$

$$
X_{A}-(k-i) \frac{n X_{A}-X_{B}}{k-1}<i \frac{X_{A}}{k-1} \leq X_{A}-(k-1-i) \frac{n X_{A}-X_{B}}{k-1} .
$$

Thus, given that player $B$ is following the equilibrium strategy, player $A$ 's allocation of $i \frac{X_{A}}{k-1}$ to an all-pay auction yields the expected payoff

$$
\frac{v i\left(\frac{2}{n}-\frac{X_{B}-X_{A}(n-2)}{v}\right)}{k}
$$

for each $i=0, \ldots, k-1$. Similarly, player $A$ 's remaining resources $(k-1-i) \frac{X_{A}}{k-1}$ have expected payoff of

$$
\frac{v(k-1-i)\left(\frac{2}{n}-\frac{X_{B}-X_{A}(n-2)}{v}\right)}{k} .
$$

Thus, for each $i=0, \ldots, k-1$ player $A$ 's allocation of $\left((k-1-i) \frac{X_{A}}{k-1}, i \frac{X_{A}}{k-1}\right)$ has an expected payoff of

$$
\frac{v(k-1)\left(\frac{2}{n}-\frac{X_{B}-X_{A}(n-2)}{v}\right)}{k} .
$$

Lastly, we consider player A's expected payoff from the uniform randomization between the mass points. Given that player $B$ is following the equilibrium strategy, the payoff to player $A$ for any allocation in which no all-pay auction is allocated more than $X_{B}-X_{A}(n-1)$ is zero. Similarly, if, for any $0<\varepsilon \leq \frac{n X_{A}-X_{B}}{k-1}$ and for some $i=$

8 For the remaining case that $i=0,0<X_{B}-X_{A}(n-1)$. 
$1, \ldots, k-2,{ }^{9}$ player $A$ allocates $X_{A}-(k-1-i) \frac{n X_{A}-X_{B}}{k-1}+\varepsilon$ to an all-pay auction the expected payoff for that all-pay auction is $v(i+1)\left(\frac{2}{n}-\frac{X_{B}-X_{A}(n-2)}{v}\right) / k$. Player $A$ 's remaining resources are $(k-1-i) \frac{n X_{A}-X_{B}}{k-1}-\varepsilon$ and

$$
(k-1-i) \frac{n X_{A}-X_{B}}{k-1}-\varepsilon \leq X_{A}-(i+1) \frac{n X_{A}-X_{B}}{k-1}
$$

since, from the definition of $k, n X_{A}-X_{B} \leq X_{A} \frac{k-1}{k}$. If player $A$ allocates all of her remaining resources to a single all-pay auction the maximum expected payoff for that all-pay auction is $v(k-i-2)\left(\frac{2}{n}-\frac{X_{B}-X_{A}(n-2)}{v}\right) / k$. Thus, for player $A$ any feasible allocation of force in which only 1 or 2 all-pay auctions receive a strictly positive level of force has a maximum expected payoff of $v(k-1)\left(\frac{2}{n}-\frac{X_{B}-X_{A}(n-2)}{v}\right) / k$. In addition, since the step size between each mass point in player $B$ 's equilibrium strategy is $\frac{n X_{A}-X_{B}}{k-1}$, player $B$ 's minimal mass point is at $X_{B}-X_{A}(n-1) \geq \frac{n X_{A}-X_{B}}{k-1}$, and each mass point has the same weight, player $A$ cannot achieve a higher expected payoff from dividing these remaining resources among more than one all-pay auction. Thus, given that player $B$ is following the equilibrium strategy, the maximum expected payoff to player $A$ for an arbitrary strategy $\mathbf{x} \in \mathfrak{B}_{A}$ is

$$
\sum_{j=1}^{n}\left[v F_{B}^{j}\left(x_{j}\right)-x_{j}\right] \leq \frac{v(k-1)\left(\frac{2}{n}-\frac{X_{B}-X_{A}(n-2)}{v}\right)}{k} .
$$

The argument for player $B$ is symmetric.

This completes the proof of Theorem 3 .

\section{Appendix C}

Subject to the constraint that there exist sufficient $n$-variate distribution functions, Theorems 1,2 , and 4 characterize the unique sets of equilibrium univariate marginal distribution functions for their respective parameter ranges.

For Theorems 1 and 2 Roberson (2006) demonstrates the existence of such $n$-variate distribution functions. This Appendix establishes the existence of sufficient $n$-variate distributions for the Theorem 4 parameter range.

${ }^{9}$ For the remaining case that $i=k-1$, player $A$ 's payoff from allocating all $X_{A}$ to a given all-pay auction is the same as if player $A$ allocates $X_{A}-\frac{n X_{A}-X_{B}}{k-1}+\varepsilon$ to the all-pay auction. This follows from the tie-breaking rule and the fact that in this case player $A$ 's remaining resources are $\frac{n X_{A}-X_{B}}{k-1}-\varepsilon$, and $\frac{n X_{A}-X_{B}}{k-1}-\varepsilon<X_{B}-X_{A}(n-1)$, for all admissible $k$ and $\varepsilon>0$, so that the payoff from player $A$ 's remaining resources is 0 . 
Theorem 5 For each unique set of equilibrium univariate marginal distribution functions, $\left\{F_{i}^{j}\right\}_{j=1}^{n}$, characterized in Theorem 4, there exists an n-copula, $C$, such that the support of the n-variate distribution function $C\left(F_{i}^{1}\left(x^{1}\right), \ldots, F_{i}^{n}\left(x^{n}\right)\right)$ is contained in $\mathfrak{B}_{i}$.

We begin with the proof for player $A$. The construction of a sufficient $n$-variate distribution function for player $\mathrm{A}$ and $X_{A} \geq \frac{v}{n}$ is outlined as follows (recall that in the Theorem 4 parameter region $X_{A}<\frac{2 v}{n}$ ). The remaining case that $X_{A}<\frac{v}{n}$ is addressed directly following this case.

1. Player $A$ selects $n-2$ of the all-pay auctions, each all-pay auction chosen with equal probability, and provides zero resources to those all-pay auctions.

2. On the remaining 2 all-pay auctions, player $A$ randomizes uniformly on three line segments: (i) $\left\{\left(x_{1}, x_{2}\right) \mid x_{1}+x_{2}=2 X_{A}-\frac{2 v}{n}\right\}$, (ii) $\left\{\left(x_{1}, x_{2}\right) \mid x_{1}=0\right.$ and $2 X_{A}-\frac{2 v}{n} \leq$ $\left.x_{2} \leq X_{A}\right\}$, and (iii) $\left\{\left(x_{1}, x_{2}\right) \mid x_{2}=0\right.$ and $\left.2 X_{A}-\frac{2 v}{n} \leq x_{1} \leq X_{A}\right\}$. This support is shown in Panel (ii) of Figure 2, and this randomization is discussed in greater detail directly following this outline.

3. There are ${ }_{n} C_{2}$ ways of dividing the $n$ all-pay auctions into disjoint subsets such that $n-2$ all-pay auctions receive zero resources with probability 1 and 2 all-pay auctions involve randomizations of resources as in point 2 . The $n$-variate distribution function formed by placing probability $\left[{ }_{n} C_{2}\right]^{-1}$ on each of these $n$-variate distribution functions has univariate marginal distribution functions which each have a mass point of $\left(1-\frac{X_{A}}{v}\right)$ at 0 and randomize uniformly on $\left(0, X_{A}\right]$ with the remaining mass.

The pivotal step in this construction is point 2. Let $x_{i}$ denote the allocation of resources to all-pay auction $i \in\{1,2\}$. Consider the support of a bivariate distribution function, $F$, for $x_{1}$ and $x_{2}$ which uniformly places mass $1-\frac{n X_{A}}{2 v}$ on each of the two following line segments

$$
\begin{aligned}
& \left\{\left(x_{1}, x_{2}\right) \mid x_{1}=0 \text { and } 2 X_{A}-\frac{2 v}{n} \leq x_{2} \leq X_{A}\right\} \\
& \left\{\left(x_{1}, x_{2}\right) \mid x_{2}=0 \text { and } 2 X_{A}-\frac{2 v}{n} \leq x_{1} \leq X_{A}\right\}
\end{aligned}
$$

and uniformly places the remaining mass, $\frac{n X_{A}}{v}-1$, on the line segment

$$
\left\{\left(x_{1}, x_{2}\right) \mid x_{1}+x_{2}=2 X_{A}-\frac{2 v}{n}\right\}
$$

This support is shown in Panel (ii) of Figure 2. 


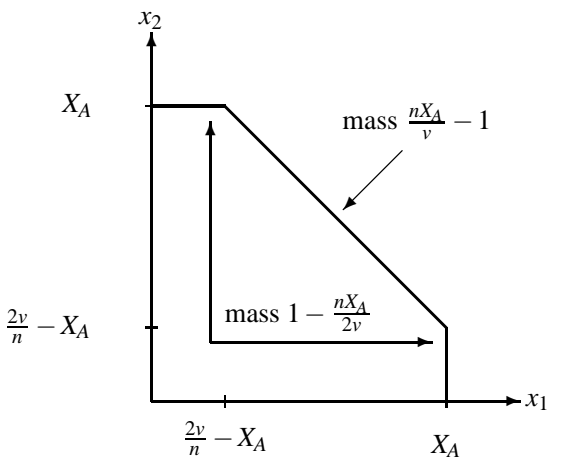

(i) Player B

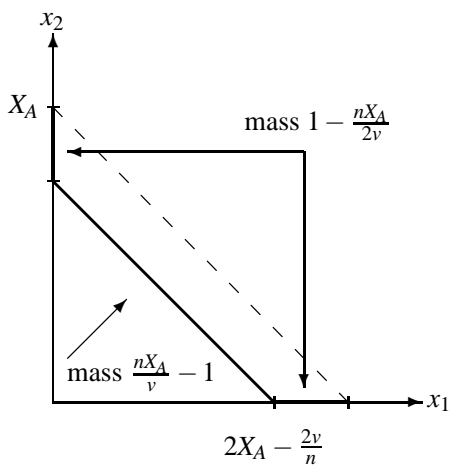

(ii) Player A

Fig. 2 Support of players' bivariate distributions $\left(\left(\bar{X}_{A} / \bar{X}_{B}\right)<(2 / n), X_{A}>(v / n)\right.$ and $\left.X_{B}>(n-2) X_{A}+(2 v / n)\right)$

In the expression for this bivariate distribution function we will use the following notation.

$$
\begin{aligned}
& \text { R1: }\left\{\left(x_{1}, x_{2}\right) \in\left[0,2 X_{A}-\frac{2 v}{n}\right]^{2}\right\} \\
& \text { R2: }\left\{\left(x_{1}, x_{2}\right) \in\left[2 X_{A}-\frac{2 v}{n}, X_{A}\right] \times\left[0,2 X_{A}-\frac{2 v}{n}\right]\right\} \\
& \text { R3: }\left\{\left(x_{1}, x_{2}\right) \in\left[0,2 X_{A}-\frac{2 v}{n}\right] \times\left[2 X_{A}-\frac{2 v}{n}, X_{A}\right]\right\} \\
& \text { R4: }\left\{\left(x_{1}, x_{2}\right) \in\left(2 X_{A}-\frac{2 v}{n}, X_{A}\right]^{2}\right\}
\end{aligned}
$$

The bivariate distribution function for $x_{1}, x_{2}$ is given by

$$
F\left(x_{1}, x_{2}\right)= \begin{cases}\left(\frac{n}{2 v}\right) \max \left\{x_{1}+x_{2}-2 X_{A}+\frac{2}{v n}, 0\right\} & \text { if }\left(x_{1}, x_{2}\right) \in \mathrm{R} 1 \\ \left(1-\frac{n X_{A}}{v}\right)+\frac{n x_{1}}{2 v}+\frac{n x_{2}}{2 v} & \text { if }\left(x_{1}, x_{2}\right) \in \mathrm{R} 2 \cup \mathrm{R} 3 \cup \mathrm{R} 4\end{cases}
$$

The univariate marginal distributions are given by $F\left(x_{1}, X_{A}\right)=\left(1-\frac{n X_{A}}{2 v}\right)+\frac{n x_{1}}{2 v}$ and $F\left(X_{A}, x_{2}\right)=\left(1-\frac{n X_{A}}{2 v}\right)+\frac{n x_{2}}{2 v}$. To see that $F$ provides the necessary univariate marginal distributions, observe that given the randomization outlined above player A allocates zero resources to each all-pay auction $j$ with probability $\frac{n-2}{n}+\frac{2}{n}\left(1-\frac{n X_{A}}{2 v}\right)=\left(1-\frac{X_{A}}{v}\right)$ and randomizes uniformly over the interval $\left(0, X_{A}\right]$ with the remaining mass.

If $X_{A}<\frac{v}{n}$, then player $A$ allocates zero resources to $n-1$ of the all-pay auctions and provides a random level of resources in the one remaining all-pay auction. In this one remaining all-pay auction player $\mathrm{A}$ has a mass point of $\left(1-\frac{n X_{A}}{v}\right)$ at 0 and randomizes uniformly over the interval $\left[0, X_{A}\right]$ with the remaining mass.

The proof for player B is similar. The construction of a sufficient $n$-variate distribution function for player B and $X_{A} \geq \frac{v}{n}$ is outlined as follows. In the Theorem 4 parameter region $X_{B} \geq \min \left\{n X_{A},(n-2) X_{A}+(2 v / n)\right\}$. If $X_{A} \geq \frac{v}{n}$ then $X_{B} \geq(n-2) X_{A}+(2 v / n)$. The remaining case that $X_{A}<\frac{v}{n}$ and $X_{B} \geq n X_{A}$ is addressed directly following this case. 
1. Player B selects $n-2$ of the all-pay auctions, each all-pay auction chosen with equal probability, and allocates $X_{A}$ to each of those all-pay auctions.

2. On the remaining 2 all-pay auctions, player $B$ randomizes uniformly on three line segments: (i) $\left\{\left(x_{1}, x_{2}\right) \mid x_{1}+x_{2}=\frac{2 v}{n}\right\}$, (ii) $\left\{\left(x_{1}, x_{2}\right) \mid x_{1}=X_{A}\right.$ and $\left.0 \leq x_{2} \leq \frac{2 v}{n}-X_{A}\right\}$, and (iii) $\left\{\left(x_{1}, x_{2}\right) \mid x_{2}=X_{A}\right.$ and $\left.0 \leq x_{1} \leq \frac{2 v}{n}-X_{A}\right\}$. This support is shown in Panel (i) of Figure 2, and this randomization is discussed in greater detail directly following this outline.

3. There are ${ }_{n} C_{2}$ ways of dividing the $n$ all-pay auctions into disjoint subsets such that $n-2$ all-pay auctions receive $X_{A}$ with probability 1 and 2 all-pay auctions involve randomizations of force as in point 2 . The $n$-variate distribution function formed by placing probability $\left[{ }_{n} C_{2}\right]^{-1}$ on each of these $n$-variate distribution functions has univariate marginal distribution functions which each have a mass point of $\left(1-\frac{X_{A}}{v}\right)$ at $X_{A}$ and randomize uniformly on $\left[0, X_{A}\right)$ with the remaining mass.

The pivotal step in this construction is again point 2. Let $x_{i}$ denote the allocation to allpay auction $i \in\{1,2\}$. Consider the support of a bivariate distribution function, $F$, for $x_{1}$ and $x_{2}$ which uniformly places mass $1-\frac{n X_{A}}{2 v}$ on each of the two following line segments

$$
\begin{aligned}
& \left\{\left(x_{1}, x_{2}\right) \mid x_{1}=X_{A} \text { and } 0 \leq x_{2} \leq \frac{2 v}{n}-X_{A}\right\} \\
& \left\{\left(x_{1}, x_{2}\right) \mid x_{2}=X_{A} \text { and } 0 \leq x_{1} \leq \frac{2 v}{n}-X_{A}\right\}
\end{aligned}
$$

and uniformly places the remaining mass, $\frac{n X_{A}}{v}-1$, on the line segment

$$
\left\{\left(x_{1}, x_{2}\right) \mid x_{1}+x_{2}=\frac{2 v}{n}\right\}
$$

This support is shown in Panel (i) of Figure 2.

The bivariate distribution function for $x_{1}, x_{2}$ is given by

$$
F\left(x_{1}, x_{2}\right)= \begin{cases}\left(\frac{n}{2 v}\right) \max \left\{x_{1}+x_{2}-\frac{2}{v n}, 0\right\} & \text { if }\left(x_{1}, x_{2}\right) \in\left[0, X_{A}\right)^{2} \\ \frac{n x_{1}}{2 v} & \text { if } x_{2}=X_{A}, x_{1} \in\left[0, X_{A}\right) \\ \frac{n x_{2}}{2 v} & \text { if } x_{1}=X_{A}, x_{2} \in\left[0, X_{A}\right) \\ 1 & \text { if } x_{1}, x_{2} \geq X_{A}\end{cases}
$$

Following from the arguments given above for player A, it follows that $F$ provides the necessary univariate marginal distributions for all-pay auctions 1 and 2 .

If $X_{A}<\frac{v}{n}$ and $X_{B} \geq n X_{A}$, then player B allocates $X_{A}$ to $n-1$ of the all-pay auctions and provides a random level of resources in the one remaining all-pay auction. In this one remaining all-pay auction player A has a mass point of $\left(1-\frac{n X_{A}}{v}\right)$ at $X_{A}$ and randomizes uniformly over the interval $\left[0, X_{A}\right)$ with the remaining mass. 
This completes the proof of the existence of sufficient $n$-variate distributions for the Theorem 4 parameter range.

\section{References}

1. Baye, M. R., Kovenock, D., De Vries, C. G.: The all-pay auction with complete information. Economic Theory 8, 291-305 (1996)

2. Borel, E.: La théorie du jeu les équations intégrales à noyau symétrique. Comptes Rendus de l'Académie 173, 1304-1308 (1921); English translation by Savage, L.: The theory of play and integral equations with skew symmetric kernels. Econometrica 21, 97-100 (1953)

3. Che, Y. K., Gale, I. L.: Caps on political lobbying. American Economic Review 88, 643-651 (1998)

4. Golman, R., Page S. E.: General Blotto: games of strategic allocative mismatch. University of Michigan, mimeo (2006)

5. Gross, O., Wagner, R.: A continuous Colonel Blotto game. RAND Corporation RM-408 (1950)

6. Hart, S.: Discrete Colonel Blotto and general lotto games. International Journal of Game Theory 36, 441-460 (2008)

7. Hillman, A. L., Riley, J. G.: Politically contestable rents and transfers. Economics and Politics 1, 17-39 (1989)

8. Kovenock, D., Roberson B.: Coalitional Colonel Blotto games with application to the economics of alliances. Purdue University, mimeo (2007)

9. Kvasov, D.: Contests with limited resources. Journal of Economic Theory 136, 738748 (2007)

10. Laslier, J. F.: How two-party competition treats minorities. Review of Economic Design 7, 297-307 (2002)

11. Laslier, J. F., Picard, N.: Distributive politics and electoral competition. Journal of Economic Theory 103, 106-130 (2002)

12. Roberson, B.: The Colonel Blotto game. Economic Theory 29, 1-24 (2006)

13. Roberson, B.: Pork-barrel politics, targetable policies, and fiscal federalism. Journal of the European Economic Association 6, (2008)

14. Weinstein, J.: Two notes on the Blotto game. Northwestern University, mimeo (2005) 


\section{CESifo Working Paper Series}

for full list see www.cesifo-group.org/wp

(address: Poschingerstr. 5, 81679 Munich, Germany, office@cesifo.de)

2317 Michael Hofmann, Gerhard Kempkes and Helmut Seitz, Demographic Change and Public Sector Budgets in a Federal System, May 2008

2318 Paul De Grauwe, Macroeconomic Modeling when Agents are Imperfectly Informed, June 2008

2319 Johann K. Brunner and Susanne Pech, Optimum Taxation of Inheritances, June 2008

2320 Thomas Eichner and Marco Runkel, Corporate Income Taxation of Multinationals in a General Equilibrium Model, June 2008

2321 Rainald Borck and Matthias Wrede, Subsidies for Intracity and Intercity Commuting, June 2008

2322 Patricia Apps and Ray Rees, Testing the Pareto Efficiency of Household Resource Allocations, June 2008

2323 Amihai Glazer, Vesa Kanniainen and Panu Poutvaara, Firms' Ethics, Consumer Boycotts, and Signalling, June 2008

2324 Claudia M. Buch, Jörg Döpke and Kerstin Stahn, Great Moderation at the Firm Level? Unconditional vs. Conditional Output Volatility, June 2008

2325 Helmuth Cremer, Philippe De Donder, Dario Maldonado and Pierre Pestieau, Forced Saving, Redistribution and Nonlinear Social Security Schemes, June 2008

2326 M. Hashem Pesaran and Paolo Zaffaroni, Optimal Asset Allocation with Factor Models for Large Portfolios, June 2008

2327 Harald Badinger and Peter Egger, Horizontal versus Vertical Interdependence in Multinational Activity, June 2008

2328 Jan K. Brueckner and Harris Selod, A Theory of Urban Squatting and Land-Tenure Formalization in Developing Countries, June 2008

2329 Paolo M. Panteghini, Corporate Debt, Hybrid Securities and the Effective Tax Rate, June 2008

2330 Guglielmo Maria Caporale, Juncal Cuñado and Luis A. Gil-Alana, Modelling Long-Run Trends and Cycles in Financial Time Series Data, June 2008

2331 Avi Ben-Bassat and Momi Dahan, Social Identity and Voter Turnout, June 2008 
2332 Martin R. West and Ludger Wößmann, "Every Catholic Child in a Catholic School”: Historical Resistance to State Schooling, Contemporary Private Competition, and Student Achievement across Countries, June 2008

2333 Erkki Koskela and Panu Poutvaara, Outsourcing and Labor Taxation in Dual Labor Markets, June 2008

2334 Philippe Choné and Laurent Linnemer, Optimal Litigation Strategies with Signaling and Screening, June 2008

2335 Albert Solé-Ollé and Pilar Sorribas-Navarro, Does Partisan Alignment Affect the Electoral Reward of Intergovernmental Transfers?, June 2008

2336 Antonio Cabrales and Piero Gottardi, Markets for Information: Of Inefficient Firewalls and Efficient Monopolies, June 2008

2337 Sumon Majumdar and Sharun W. Mukand, The Leader as Catalyst - on Leadership and the Mechanics of Institutional Change, June 2008

2338 Ulrich Hange, Tax Competition, Elastic Labor Supply, and Growth, June 2008

2339 Guy Laroque and Bernard Salanié, Does Fertility Respond to Financial Incentives?, June 2008

2340 Adriano Paggiaro, Enrico Rettore and Ugo Trivellato, The Effect of Extending the Duration of Eligibility in an Italian Labour Market Programme for Dismissed Workers, June 2008

2341 Helmut Seitz, Minimum Standards, Fixed Costs and Taxing Autonomy of Subnational Governments, June 2008

2342 Robert S. Chirinko, Leo de Haan and Elmer Sterken, Asset Price Shocks, Real Expenditures, and Financial Structure: A Multi-Country Analysis, July 2008

2343 Wolfgang Leininger, Evolutionarily Stable Preferences in Contests, July 2008

2344 Hartmut Egger and Udo Kreickemeier, Fairness, Trade, and Inequality, July 2008

2345 Ngo Van Long and Bodhisattva Sengupta, Yardstick Competition, Corruption, and Electoral Incentives, July 2008

2346 Florian Baumann, Employment Protection: The Case of Limited Enforceability, July 2008

2347 Alessandro Balestrino, Cinzia Ciardi and Claudio Mammini, On the Causes and Consequences of Divorce, July 2008

2348 Dirk Schindler and Benjamin Weigert, Insuring Educational Risk: Opportunities versus Income, July 2008 
2349 Lammertjan Dam and Ben J. Heijdra, The Environmental and Macroeconomic Effects of Socially Responsible Investment, July 2008

2350 Avner Greif, Contract Enforcement and Institutions among the Maghribi Traders: Refuting Edwards and Ogilvie, July 2008

2351 Helmuth Cremer, Philippe De Donder, Dario Maldonado and Pierre Pestieau, Habit Formation and Labor Supply, July 2008

2352 Francesco Menoncin and Paolo M. Panteghini, The Johansson-Samuelson Theorem in General Equilibrium: A Rebuttal, July 2008

2353 Michael Kaganovich and Itzhak Zilcha, Alternative Social Security Systems and Growth, July 2008

2354 Keith Blackburn, Kyriakos C. Neanidis and M. Emranul Haque, Corruption, Seigniorage and Growth: Theory and Evidence, July 2008

2355 Edward Castronova, A Test of the Law of Demand in a Virtual World: Exploring the Petri Dish Approach to Social Science, July 2008

2356 Harald Badinger and Peter Egger, GM Estimation of Higher-Order Spatial Autoregressive Processes in Cross-Section Models with Heteroskedastic Disturbances, July 2008

2357 Wolfgang Buchholz and Jan Schumacher, Discounting the Long-Distant Future: A Simple Explanation for the Weitzman-Gollier-Puzzle, July 2008

2358 Luca Anderlini, Leonardo Felli and Alessandro Riboni, Statute Law or Case Law?, July 2008

2359 Guglielmo Maria Caporale, Davide Ciferri and Alessandro Girardi, Are the Baltic Countries Ready to Adopt the Euro? A Generalised Purchasing Power Parity Approach, July 2008

2360 Erkki Koskela and Ronnie Schöb, Outsourcing of Unionized Firms and the Impacts of Labour Market Policy Reforms, July 2008

2361 Francisco Alvarez-Cuadrado and Ngo Van Long, A Permanent Income Version of the Relative Income Hypothesis, July 2008

2362 Gabrielle Demange, Robert Fenge and Silke Uebelmesser, Financing Higher Education and Labor Mobility, July 2008

2363 Alessandra Casarico and Alessandro Sommacal, Labor Income Taxation, Human Capital and Growth: The Role of Child Care, August 2008

2364 Antonis Adam, Manthos D. Delis and Pantelis Kammas, Fiscal Decentralization and Public Sector Efficiency: Evidence from OECD Countries, August 2008 
2365 Stefan Voigt, The (Economic) Effects of Lay Participation in Courts - A Cross-Country Analysis, August 2008

2366 Tobias König and Andreas Wagener, (Post-)Materialist Attitudes and the Mix of Capital and Labour Taxation, August 2008

2367 Ximing Wu, Andreas Savvides and Thanasis Stengos, The Global Joint Distribution of Income and Health, August 2008

2368 Alejandro Donado and Klaus Wälde, Trade Unions Go Global!, August 2008

2369 Hans Gersbach and Hans Haller, Exit and Power in General Equilibrium, August 2008

2370 Jan P.A.M. Jacobs and Jan-Egbert Sturm, The Information Content of KOF Indicators on Swiss Current Account Data Revisions, August 2008

2371 Oliver Hülsewig, Johannes Mayr and Timo Wollmershäuser, Forecasting Euro Area Real GDP: Optimal Pooling of Information, August 2008

2372 Tigran Poghosyan and Jakob de Haan, Determinants of Cross-Border Bank Acquisitions in Transition Economies: A Latent Class Analysis, August 2008

2373 David Anthoff and Richard S.J. Tol, On International Equity Weights and National Decision Making on Climate Change, August 2008

2374 Florian Englmaier and Arno Schmöller, Reserve Price Formation in Online Auctions, August 2008

2375 Karl Farmer, Birgit Friedl and Andreas Rainer, Effects of Unilateral Climate Policy on Terms of Trade, Capital Accumulation, and Welfare in a World Economy, August 2008

2376 Monika Bütler, Stefan Staubli and Maria Grazia Zito, The Role of the Annuity’s Value on the Decision (Not) to Annuitize: Evidence from a Large Policy Change, August 2008

2377 Inmaculada Martínez-Zarzoso, The Impact of Urbanization on $\mathrm{CO}_{2}$ Emissions: Evidence from Developing Countries, August 2008

2378 Brian Roberson and Dmitriy Kvasov, The Non-Constant-Sum Colonel Blotto Game, August 2008 